\title{
Un viaje sanador
}

Volumen 5 N. ${ }^{\circ} 42$

enero - junio de 2017

ISSN: 0122-4328

ISSN-E: 2619-6069

pp. $117-124$

\author{
A Journey of Healing $\quad \begin{aligned} & \text { Uma viagem } \\ & \text { sanadora }\end{aligned}$
}

\section{Dalila Esperanza Castillo Sánchez* \\ Ingrid Astrid Rubio Parra** \\ Nelson Javier Velosa Cruz***}

Fecha de recepción: 28-08-2015

Fecha de aprobación: 03-03-2017

\section{PARA CITAR ESTE ARTÍCULO}

Castillo, D., Rubio, I., Velosa, N. (2018). Un viaje sanador. Nodos y Nudos, 42, 117-124.

Maestra de Lengua Castellana del Instituto Pedagógico Nacional. dalilahope@yahoo.com

* Maestra de Ética del Instituto Pedagógico Nacional. negrita1120@yahoo.com

** Maestro de Tecnología del Instituto Pedagógico Nacional. nelsonjsc@gmail.com 


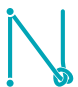

Volumen 5 N. ${ }^{\circ} 42$ enero - junio de 2017 ISSN: 0122-4328 ISSN-E: 2619-6069 pp. $117-124$

\section{RESUMEN}

Este artículo presenta una reflexión de tres maestros del Instituto Pedagógico Nacional sobre la relevancia que tienen los viajes expedicionarios en la vida de los maestros del país, luego de la participación en un Proyecto de Sistematización del IDEP, en el marco del componente de Cualificación Docente, que permitió el acompañamiento de experiencias in situ, generando un intercambio de experiencias entre colectivos de docentes de colegios de Bogotá y experiencias de colegios de otras regiones del país. La reflexión se da en torno a la crónica de un viaje realizado a la ciudad de Medellín donde el intercambio permitió a los maestros "sanar» a través del intercambio pedagógico y cultural.

Palabras clave: viaje sanador; intercambio de experiencias pedagógicas; práctica pedagógica; trabajo por proyectos

\section{ABSTRACT}

This paper presents a reflection by three teachers from Instituto Pedagógico Nacional about the relevance of expeditionary trips in the life of the teachers of the country, after taking part in an IDEP Systematization Project in the context of the Teacher Qualification component, which allowed following on-site experiences, thus generating an exchange of experiences between groups of teachers from schools in Bogotá and schools in other regions of the country. The reflection is about the chronicles of a trip made to Medellin, where the exchange allowed the teachers to "heal" through the pedagogical and cultural exchange.

Keywords: healing journey; exchange of pedagogical experiences; pedagogical practice; project work

\section{RESUMO}

Este artigo apresenta uma reflexão realizada por três professores de Instituto Pedagógico Nacional sobre a relevância das viagens expedicionárias na vida dos professores do país depois de sua participação em um Projeto de Sistematização do IDEP, no marco do componente de Qualificação Docente, que possibilitou o seguimento de experiências in situ, gerando um intercâmbio de experiências entre coletivos de professores de colégios de Bogotá e experiências de colégios de outras regiões do país. A reflexão acontece em torno à crónica de uma viagem realizada à cidade de Medellín, onde o intercâmbio permitiu aos professores "sanar" através do intercâmbio pedagógico e cultural.

Palavras-chave: viagem sanadora; intercâmbio de experiências pedagógicas; prática pedagógica; trabalho por projetos 
A pesar de la fría mañana del miércoles 6 de mayo de 2015, emprendimos un cálido y motivante viaje a la ciudad de Medellín. Al hombro, llevábamos tres mochilas llenas de grandes expectativas acerca de las escuelas que íbamos a conocer en esa hermosa ciudad. Imaginábamos que los niños y los maestros de esas escuelas, al igual que nosotros, podian estar ansiosos de intercambiar algunas experiencias pedagógicas. El cierre del aeropuerto de Rionegro aumentó la ansiedad y la preocupación por no llegar a tiempo para compartir con el grupo de maestros que nos estaba esperando.

Al llegar, nos recibió el calor de la ciudad y de la gente paisa. Ya estábamos listos para compartir un delicioso café con los maestros de Medellín. Teníamos, gracias al trabajo entre el IDEP ${ }^{1}$ y la UPN, la oportunidad ideal para valorar y enriquecer aún más nuestro quehacer con los niños. Sin embargo, se percibía también en el ambiente la tensión de los maestros pues estábamos en pleno Paro Nacional de Educadores, lo que por supuesto no permitió llevar a cabo algunas de las actividades programadas. No obstante, decidimos aprovechar el tiempo y compartir con algunos compañeros de otros colegios de Bogotá que viajaban con nosotros, con quienes nos encontrábamos por primera vez. Nos sorprendió que muchos directivos acompañaban a los maestros de aula. Los profesores que trabajaban como rectores o como coordinadores conocían muy bien los proyectos que se realizaban en sus colegios. Fue clara para nosotros la importancia de fortalecer las relaciones entre directivos y maestros.

\section{El Parque Biblioteca España}

Medellín es una ciudad tan dinámica y atractiva que no nos fue difícil iniciar nuestro viaje para conocerla, al fin y al cabo para nosotros era otra nueva experiencia para vivir. Iniciamos nuestro recorrido visitando el Parque Biblioteca España. Alli se realizan diversas actividades con las que se busca integrar a la comunidad, cambiando el concepto tradicional de biblioteca, abandonando el formalismo tradicional de muchas

1 El Instituto para la Innovación y el Desarrollo Pedagógico de Bogotá (IDEP) es una entidad del sector educativo de la Alcaldía de Bogotá dedicado a la investigación y a la promoción del trabajo pedagógico de los maestros de la ciudad. En el año 2015 el Instituto adelantó, en convenio con la Universidad Pedagógica Nacional (UPN), que tenía una amplia experiencia en Expedición Pedagógica, el Proyecto "Cualificación de maestras y maestros en ejercicio. Intercambio de experiencias pedagógicas significativas", en el marco del cual se vivió esta experiencia. bibliotecas, con el fin de acercarla al concepto de parque. Así, el lugar se convierte en un espacio donde la comunidad entera, sin distingo de edad, estrato, raza o género, se integra a actividades lúdico-recreativas, culturales y académicas. Con ello en el parque biblioteca se reconoce la voz de los habitantes y, además, se hacen visibles algunas tradiciones y habilidades manuales de la gente del barrio. En definitiva, desde su diseño y su construcción, se trata de un lugar innovador e inspirador para la labor del maestro como artesano de los sueños de aquellos pequeños que tenemos frente a nosotros cada mañana en nuestras escuelas.

Al terminar nuestro recorrido por el Parque Biblioteca España nos dirigimos a hacer un corto recorrido por el parque Berrío. Alli también encontramos una gran riqueza cultural y un espacio urbano en el que el rebusque se manifiesta de infinitas formas. Los vendedores ambulantes, los habitantes de calle, la gente del común y los voceadores de diversos negocios creaban un ambiente caótico que nos generó cierta desconfianza. Caminamos cuidando el bolsillo de aquellos que nos rodeaban y nos hacian sentir que no estábamos en un lugar seguro, sino en medio del bullicio y las carreras de todos. En realidad nos había cogido la noche y solo estábamos caminando sin rumbo fijo. Sin embargo, nada diferente a lo que sucede en la ciudad de Bogotá, donde el caos forma parte de nuestro entorno.

\section{El Centro de Innovación del Maestro y los encuentros de experiencias}

El segundo dia fue aún más inspirador, pues la siguiente parada la realizamos en el Centro de Innovación del Maestro (Mova), una propuesta con sello antioqueño. mova es el sueño de los maestros, en el que se integran el maestro, la escuela y la ciudad para posibilitar la creación de prácticas educativas diversas. Es un sueño que ha sido posible gracias al apoyo económico de la Gobernación de Antioquia, en donde conocimos experiencias de formación docente de maestros del Cauca y Putumayo.

De este centro partimos al conversatorio con la profesora Ángela Maria Yepes, una de las maestras expedicionarias de Medellin, y con algunas de sus 
compañeras de la Expedición Pedagógica Nacional ${ }^{2}$, quienes nos guiaron por su propuesta, denominada "Un viaje más allá de la tiza y el tablero". Gracias a ellas aprendimos a valorar nuestras propias prácticas pedagógicas. Nos contaron de algunos de los viajes expedicionarios realizados a diferentes lugares del país para indagar acerca de experiencias e investigaciones pedagógicas, así como sobre propuestas alternativas de hacer escuela que los maestros construyen en muchos lugares de Colombia, y nos relataron los aprendizajes que estos viajes dejaron en cada una de ellas. Luego se dio paso a la presentación de algunas experiencias pedagógicas de compañeros de Bogotá. Se presentaron los trabajos de algunos colegios distritales: Santa Marta, Rodolfo Llinás, José Asunción Silva, entre otras. Además, se presentaron cuatro proyectos del Instituto Pedagógico Nacional (IPN), en dos líneas de trabajo: la de Lenguaje e infancia, y la de Tecnología. Al conocer los proyectos pudimos percibir que muchos de ellos surgen como respuestas a necesidades propias de su contexto. Algunas de estas iniciativas cuentan con el apoyo institucional para su desarrollo, mientras que otras se ven reducidas a ser proyectos individuales de los maestros. Estos últimos encuentran en convocatorias como las generadas en el viaje a Medellín oportunidades para promover sus propuestas y obtener apoyo moral y económico. Se destaca que en el IPN, en contraste con lo que sucede en otros colegios, se cuenta con un apoyo significativo por parte de las directivas para generar, poner en práctica y divulgar los procesos pedagógicos innovadores que se desarrollan con los estudiantes en el aula. El trabajo interdisciplinar, que proviene de la pedagogía por proyectos, es una oportunidad para que los maestros establezcamos un diálogo de saberes que configure nuevos rumbos de aprendizaje en nuestros estudiantes. En este sentido, el IPN se ha destacado desde décadas atrás, dando lugar a prácticas innovadoras de aula, en las que el estudiante es el centro del proyecto pedagógico y en las

\footnotetext{
2 La Expedición Pedagógica Nacional es un proyecto realizado desde 1999 a través de viajes y encuentros de maestros en los que se comparten y analizan las propuestas e innovaciones que se desarrollan en los colegios del país, con el fin de explorar la riqueza del trabajo pedagógico, de las formas de ser maestro y de hacer escuela en Colombia. La Expedición Pedagógica es hoy un movimiento intelectual y social de maestros que se vinculan en redes, grupos, colectivos y distintas expresiones para construir nuevas miradas de nuestra educación, así como propuestas de transformación y de innovación educativa.
}

que las diferentes disciplinas aportan al desarrollo de dicho proyecto.

\section{Aprendiendo con las maestras Gladys y Nohora, y con el bibliotecario Duván}

Al siguiente día continuamos nuestro caminar complementando nuestras experiencias con dos nuevas prácticas pedagógicas de esta linda ciudad: las de los colegios Sor Juana Inés de la Cruzy Alfredo CockArango. En la dinámica de presentación nos propusieron dividir el grupo para poder conocer ambas propuestas. Las dos nos llevaron a seguir valorando lo que tenemos y hacemos cada día en nuestro colegio, asi en algunas ocasiones nos parezca tan normal y no le demos mayor importancia.

El área del colegio Sor Juana Inés de la Cruz es tan grande como la de nuestra zona de juego en Jardín en el IPN. Alli asisten cinco grupos, que deben compartir el espacio disponible. Se trata de una institución pequeña, que cuenta con unos ciento ochenta estudiantes, organizados en grupos de treinta a treinta y cinco niños por salón en los cursos de la sección de primaria.

Alli nos encontramos con Gladys, maestra convencida y entregada a su grupo de estudiantes de Preescolar, a quienes escucha atentamente y quien, en muchas ocasiones, pone en práctica las sugerencias que ellos hacen para el desarrollo de las clases. En el grupo del grado Transición, en particular, los niños han ido fortaleciendo su autonomía a través de la utilización del cuerpo, combinado con la de la música y con mínimas orientaciones de la profesora. Gladys no utiliza libros de texto y aplica una pedagogía inductiva ${ }^{3}$, en la que el proceso de formación de sus pequeños estudiantes se da a través de instrucciones generales, sin mayores explicaciones. De esta manera los niños asumen responsabilidades y funciones en el desarrollo de las actividades diarias, lo cual genera una agenda diferente para cada día, construida con la participación de todos. El resultado es una organización y una dinámica de aula en la que no es necesaria la presencia perma-

\footnotetext{
3 La pedagogía inductiva también se conoce como pedagogía del descubrimiento. En ella el sujeto no aprende a partir de una información que recibe de forma pasiva, sino que, al estilo de las pedagogías activas, el aprendizaje se produce desde el descubrimiento y la apropiación de experiencias individuales.
} 
nente de la maestra, pues los estudiantes por sí solos saben lo que van a realizar durante el día.

Por otra parte, las clases de Educación Física se integran con las de Matemáticas y con las de otras asignaturas, a través de diferentes actividades que llevan a los chicos a tener un aprendizaje interdisciplinar. El buen trato entre compañeros se motiva por medio de la responsabilidad compartida en el cuidado de una mascota llamada Coquito. Esta mascota se lleva por un día a la casa de quien ha tenido un buen comportamiento durante la jornada escolar. Los niños que asumen la responsabilidad de cuidar de Coquito realizan una consulta en su familia para poder llevarlo a su casa como un invitado especial. Además, deben llevar un diario que llega con la mascota a cada casa para registrar la experiencia.

Sin embargo, el trabajo de la maestra Gladys no está coordinado con el de sus compañeras, no tiene continuidad en los grados siguientes, debido a que hay maestras que no quieren cambiar su línea tradicional de enseñanza. De cualquier forma, conocer la práctica pedagógica de esta maestra impacta y motiva a seguir experimentando nuevas metodologías de enseñanza, que fortalezcan al niño no solo en lo académico sino en la integralidad de su ser.

La otra experiencia que conocimos y que vivimos fue la del colegio Alfredo Cock Arango, donde la maestra Nohora y el joven bibliotecario Duván Gil nos compartieron sus maravillosas prácticas pedagógicas. En palabras de la profesora, se trata de prácticas que no revolucionan el mundo ni pretenden "descubrir el agua tibia", pero sí han incidido, primero en los pequeñitos de su salón, y ahora en muchos de los estudiantes de su colegio, pues ha logrado contagiar positivamente a la mayoría de los maestros y funcionarios que allí trabajan el ánimo de sanar vidas.

El cuento con los cuentos infantiles es el nombre que la maestra Nohora le dio a su experiencia. En ella, parte de una problemática que se presenta entre los estudiantes de su salón de preescolar, en su contexto, en el que la violencia es el pan de cada día, donde las fronteras invisibles que imponen los "combos"4 limitan una sencilla ida al parque, o la visita a un familiar que

4 "Combos" se refiere a los grupos de jóvenes organizados de las comunas de Medellín que están asociados a las bandas delicuenciales de la ciudad vive a tan solo unas cuadras de distancia. En estos contextos las familias son prácticamente nómadas, porque la violencia las obliga a desarraigarse continuamente de sus vecinos y amigos; allí la mayoría de los niños no cuentan con familias con papá y mamá, y el hambre es uno más de los suyos.

Una vez identificadas las vivencias de los niños sobre las cuales se propone trabajar, la maestra busca un cuento infantil que trate de dicha problemática, lo lee a los estudiantes y los lleva a relacionar lo que sucede en la historia del cuento con su contexto diario. A simple vista parece una práctica sencilla que se hace en muchos salones de clase con profesores de Lengua Castellana o Ética. Sin embargo, hay que conocer de cerca esta estrategia y la profundidad con que ha llegado a impactar la vida de estos doce niños y sus familias, la pasión y el convencimiento que tiene la profesora de lo sanadores que pueden ser los cuentos, una terapia para el alma de los chicos.

Alli es donde esta experiencia pedagógica cobra tanta fuerza, porque logra aliviar las heridas con que estos conflictos quieren oscurecer esos pequeños corazones... y alli es donde este viaje también se convierte en un viaje sanador... un viaje que nos permite recordar por qué elegimos un día convertirnos en maestros, por qué persistimos en repensar nuestras prácticas y buscar un cambio a pesar de que la sociedad desestime nuestra labor; que nos lleva a indagar por qué como maestros continuamos generando buenas -o no tan buenas- ideas para tocar la vida de nuestros estudiantes y por qué aceptamos realizar este viaje.

Por otro lado, conocimos el trabajo de Duván Gil, el bibliotecario de la institución, quien, a pesar de haber llegado tan solo hace unos pocos meses al colegio, ha puesto en marcha sus estrategias Ilamadas Lecturas gratis y Secuencias didácticas. Dichas estrategias se fundamentan en la definición de planes lectores para cada asignatura por parte de los maestros y el bibliotecario. Se busca con ello que los estudiantes y los docentes encuentren en la biblioteca un espacio idóneo para la investigación, la lectura, el análisis, la escritura y, en definitiva, el aprendizaje. Una experiencia refrescante para el proyecto de lectura, escritura y oralidad de la institución que, gracias al compromiso de Duván, ha permeado todo el currículo. 


\section{El Parque Explora}

La última parada de nuestro viaje fue en el Parque Explora, un lugar alucinante que busca ser mucho más que un simple sitio para la diversión y la distracción. En realidad alli se pueden aprender algunas cosas, y es posible construir un espacio en el que la ciudad establece una relación real, cercana y muy creativa con el conocimiento. En consecuencia con ello, el Parque abre sus puertas con el fin de que los maestros de la ciudad y de la periferia se reúnan constantemente para que las prácticas de sus aulas alimenten y enriquezcan las del parque. Biomae, Astromae y Chiquimae son algunas de las estrategias que Maestros Amigos de Explora (MAE) ha creado para que los maestros aporten a la vida del Parque Explora.Este fue un viaje que develó ante nuestros ojos nuevas formas de innovar, no solo en las prácticas de los maestros sino en los espacios y tiempos dentro de la escuela e incluso en la ciudad. Las diversas formas en que los maestros de Medellín se apropian de una ciudad completa como aula para el aprendizaje permitieron regenerar nuevos tejidos educativos que pueden contribuir a lograr transformaciones. Como maestros necesitamos sanar aquellas heridas que los obstáculos propios de nuestra profesión, como la indiferencia de las administraciones educativas, las familias y la misma sociedad, van formando en nuestro ejercicio docente. En algunas ocasiones, la monotonía, la rutina de la escuela y el cansancio del maestro también pueden ir desgastando su salud mental y física. No obstante, dichas afecciones van superándose cuando vemos en otro maestro la pasión que lleva dentro y la misma vocación que nos hizo elegir la enseñanza como nuestra labor; eso nos inyecta vitaminas de vida, oxigenando el cuerpo y el espíritu. Cuando un grupo de maestros se ha tomado el tiempo para visitarte, verte y escuchar lo que haces diariamente con tus estudiantes, puedes pensar que algo estás haciendo bien en la escuela. Definitivamente un viaje puede sanar. Puede sanar al reflejar en un colega que comparte su experiencia pedagógica a ese maestro que siempre quisimos ser, un maestro enamorado de la profesión docente, un maestro que no desiste sino que persiste, un maestro que trabaja con los recursos que tenga disponibles, no echando de menos aquellos que le faltan para convertirlo en la excusa perfecta para no cambiar su práctica pedagógica, sino un maestro que vea el vaso medio lleno, así otros lo quieran ver medio vacío. Este viaje nos sanó. Ser maestro es una opción de vida, personal e intransferible, una profesión donde construimos con el otro, por el otro y para otros; pero también es aprender a valorar nuestro ejercicio y el de nuestros compañeros. Fue un viaje que logró tocar esas fibras de nuestro espíritu que, quizás dormidas, aún anhelan que seamos maestros reflexivos, críticos y, por qué no, transformadores del quehacer de nuestro querido Instituto Pedagógico Nacional. 


\section{DIÁLOGO DEL CONOCIMIENTO}

\section{Nosotros}

Desde el momento en el que los editores de Nodos y Nudos me pidieron escribir un diálogo con el artículo "Un viaje sanador" me llené de dudas y de incertidumbre. Lo que en principio parecía ser una tarea sencilla se convirtió para mí en una labor ardua. Por una parte, debía decir muchas cosas en solo unos pocos párrafos, lo que nunca ha sido una de mis fortalezas. Por otra, enfrentaba un reto de mucha mayor envergadura: ¿cómo entrar en conversación con el testimonio de un grupo de maestros sobre el valor de una expedición pedagógica sin caer en la repetición y en los lugares comunes?

Tanto se ha dicho acerca del valor formativo de los intercambios entre maestros, tanto se ha escrito sobre la importancia de viajar para educadores dispuestos a experimentar formas alternativas de enseñanza y comprometidos con la utopía de consolidar maneras innovadoras de hacer escuela, que no tiene sentido volver, casi con insistencia dogmática, sobre el poder innovador de salir de la escuela, de visitar otros territorios, de conocer otras condiciones, o simplemente de conversar con colegas con diferentes miradas y en distintas condiciones. ¿Para qué insistir en ello si ya los mismos maestros que han experimentado el papel de expedicionarios lo han dicho con claridad incontrastable?

Lo cierto es que lo que finalmente terminen siendo los párrafos que usted lector tiene ahora entre sus manos ha sido el producto de la prueba y del error. Mejor sería utilizar el plural y decir que ha sido consecuencia de las pruebas y de los errores, porque en realidad han sido necesarios muchos ensayos para llegar solo hasta aquí. Por fortuna, en estos tiempos de escrituras digitales no es necesario romper papeles y tirar a la basura los diferentes intentos realizados; basta con borrar los archivos y enviarlos a la papelera de los computadores. Así lo he hecho en muchas ocasiones con múltiples pruebas de este texto. 


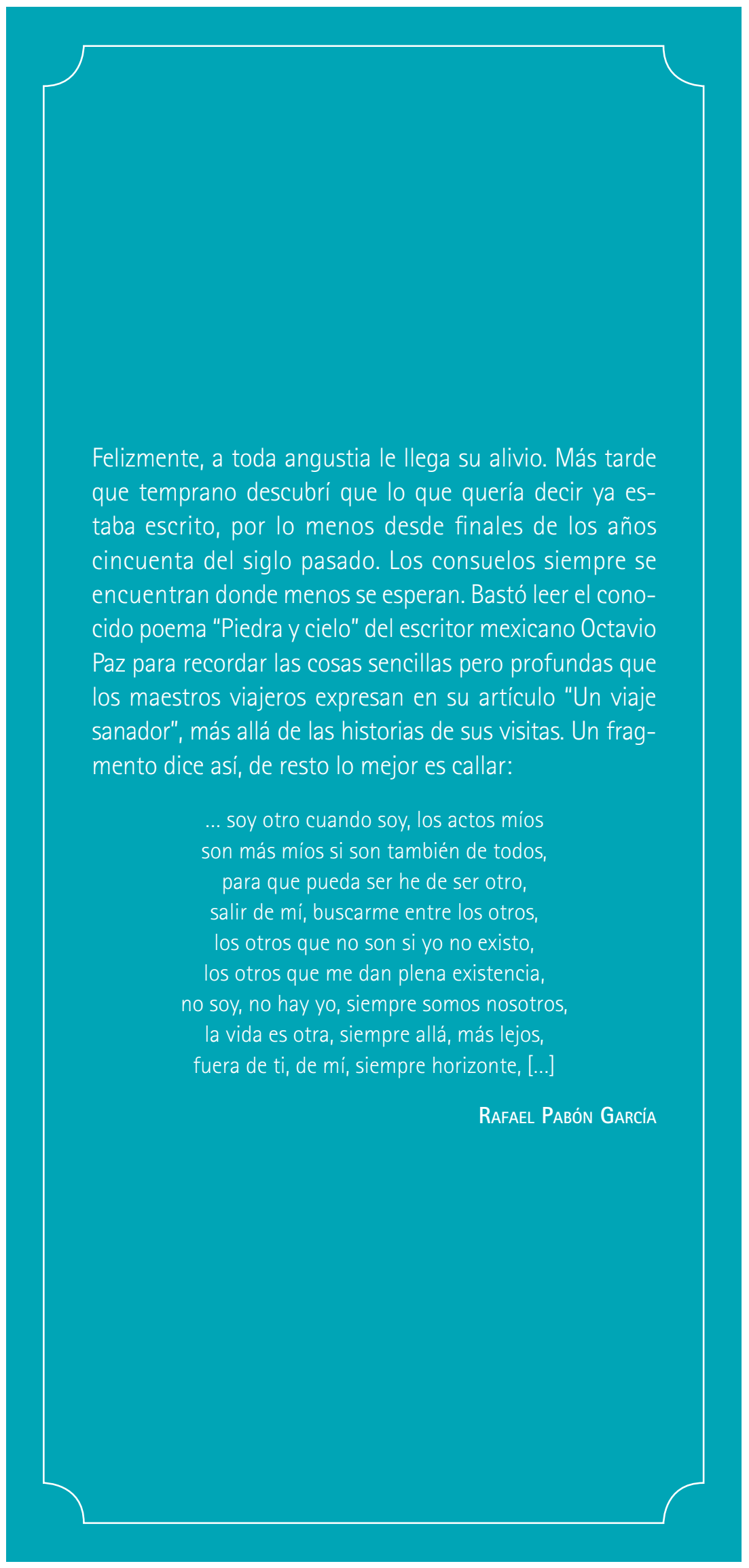

